\title{
The Influence Factors Analysis to Improve the Compatibility and the Mechanical Behavior of IPN, Gradient IPN and $\mathrm{BaTiO}_{3}$ Filled IPN
}

\author{
Yudi Guo, Dongyan Tang*, Ying Wang \\ Department of Chemistry, Harbin Institute of Technology, Harbin, China. \\ Email: *dytang@hit.edu.cn
}

Received May 15 ${ }^{\text {th }}, 2012$; revised June 20 ${ }^{\text {th }}, 2012$; accepted July 21 ${ }^{\text {st }}, 2012$

\begin{abstract}
Interpenetrating polymer network (IPN), gradient IPN and $\mathrm{BaTiO}_{3}$ filled IPN, composed of poly(ethylene glycol urethane) (PEGPU) and unsaturated polyester resin (UP) curing at room temperatures were prepared. Then the effect of soft/hard segment ratio in polyurethane (PU), component ratio of PU to UP in IPN, adding amount of $\mathrm{BaTiO}_{3}$ in filled IPN, the component sequences and interval times between each IPN for gradient IPN, on morphology and mechanical behavior of IPN and $\mathrm{BaTiO}_{3} / \mathrm{IPN}$ nanocomposites with different molecular weight of PU were studied systematically. Moreover, the investigation on the relationship between the morphologies and the mechanical properties indicated that the IPN with finer morphology exhibited an excellent consistency of the higher strengths and elongations.
\end{abstract}

Keywords: Poly(ethylene Glycol Urethane) (PEGPU); Gradient Interpenetrating Polymer Networks (Gradient IPN); $\mathrm{BaTiO}_{3}$; Morphology; Mechanical Property

\section{Introduction}

Owing to the mutual entanglement structural characteristics created by the forced compatibility and the synergism of the two networks of IPN, the mechanical properties of IPN are superior to those of ordinary polymers $[1,2]$. In particular, the maximum tensile strength of IPN can be much higher than that of its constituent polymers $[3,4]$. Therefore, many valuable systems have been made by taking advantage of the IPN structure [5,6]. Also, much efforts have been put to investigate that how to improve the mechanical properties of IPN and what is the relationship of its mechanical properties with the structures of IPN [7-9]. In our previous papers [10], two series of IPN, $\mathrm{BaTiO}_{3}$ filled IPN, and the gradient IPN have been prepared, and some properties of such materials have been investigated in details. It is found that the types and its crosslink density of polyurethane influence the morphology of IPN greatly, and consequently influence the other properties of the materials [11]. Generally, the structures and the properties of the polymeric materials are related to the molecular weight of the components [12]. However, little attention has been paid to the relation of molecular weight in IPN to the structure and properties of the materials $[13,14]$. So in the present work, a series

"Corresponding author. of $\mathrm{BaTiO}_{3}$ filled and unfilled interpenetrating polymer networks (IPN), gradient IPN composed of poly(ethylene glycol urethane) (PEGPU) and unsaturated polyester resin (UP) with different molecular weight of PU were prepared. The aim of the work is to investigate the effect of molecular weight of PU with different soft/hard segment ratio in $\mathrm{PU}$, different component ratio of $\mathrm{PU}$ to UP in IPN and different adding amount of $\mathrm{BaTiO}_{3}$ in filled IPN, and the component sequences and interval times of each IPN in the gradient IPN, on the morphology and the mechanical properties, and also to reveal the relationship between the morphologies and their mechanical properties.

\section{Experimental}

Raw materials, their pretreatment and the preparation procedures of IPN are all the same with that described previously [10]. Phase morphology of IPN and $\mathrm{BaTiO}_{3}$ filled IPN was examined on a Nippon Electron 1200-EX transmission electron microscope (TEM). The TEM detection samples were ultramicrotomed into $100 \mathrm{~nm}$ thick sections and stained in $\mathrm{OsO}_{4}$ solution (2\% by weight) for 18 - 24 hours. Morphology detection of gradient IPN was detected by Energy Dispersive X-ray (EDX) analysis through the using of the Oxford ISIS-300 spectrometer coupled with a JEOL JXA-scanning electron microscope (SEM) on the cross-section of a sample in advance to determine the 
transitional ranges in gradient IPN. Then the select gradient IPN was observed by TEM through the fracturing it in liquid nitrogen. The tensile strength, shear strength, elongation at break of IPN, gradient IPN and $\mathrm{BaTiO}_{3}$ filled IPN in small dumb-bell patterns were measured by the Nippon Shimadzu DSS-10T-S tester at room temperature at a crosshead speed of $5 \mathrm{~mm} / \mathrm{min}$ according to China Standard (GB4456-84). Data were taken as average of at least five measurements.

\section{Results and Discussion}

\subsection{The Effect of the Soft/Hard Segments Ratios with Different Molecular Weight of PEGPU in IPN}

Generally, the chemical dissimilar of the soft and hard segments in polyurethane tends to produce the incompatible structures and thus separate the product into the different phases during its formation. Varying the equivalent ratio of the isocyanate (-NCO) group to the hydroxyl (-OH) group in polyurethane is one of the effect modifying methods to improve the compatibility [15]. Figure 1 shows the tensile strength (a), the elongation at break (b), and the shear strength (c) of polyurethane with varied molar ratio of $-\mathrm{NCO} / \mathrm{-OH}$ (represented as the symbol of R). Seen from the figure, varying the $\mathrm{R}$ values and changing the complexity and/or crosslink degree of PU by using the different molecular weight (MW) of PEGPU in IPN can both modulate the product's compatibility. The strengths of the IPN with various $R$ values increased with the increase of MW of PU at first, then decreased slowly because of the decreased chain relaxation during the extension by the higher crosslink density. Since the strength of the elastomer of PU is related to the relaxation time of the mobile chain and the hard segment in PU are the main donor to the strength, so the strengths increased with the increase of the $\mathrm{R}$ at first and then decreased slowly.

The PU with lower MW can penetrate into the UP networks more easily and thus exhibit the higher compatibility. So the approximate crosslink density of PU contributes the approximate penetrating structures, further contributes the approximate compatibility. That is why the tensile strengths, the elongation and the shear strengths can all reach the relative higher values for the PEGPU1000/UP IPN.

The UP in IPN can be stained selectively by $\mathrm{RuO}_{4}$ solution and appears as the dark phase in the TEM photographs (as seen in Figure 2). The domain sizes of PEGPU1000/UP IPN are smaller than that of PEGPU600/UP IPN

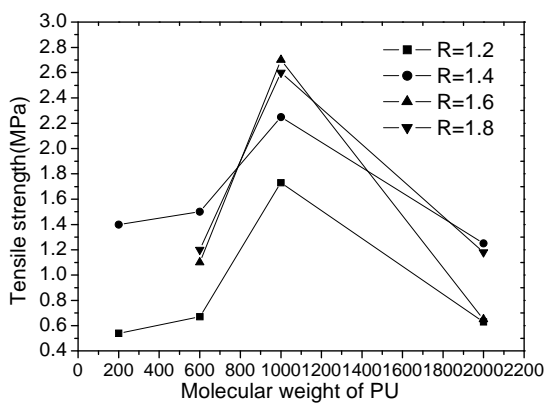

(a)

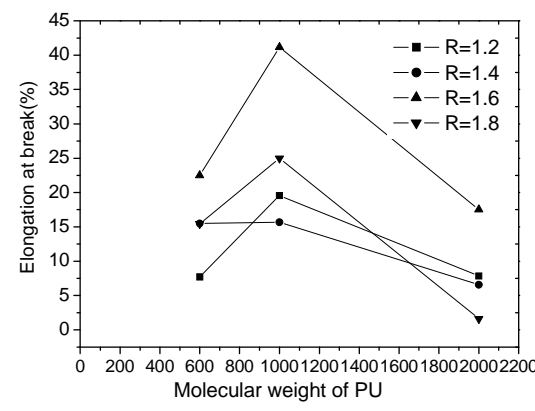

(b)

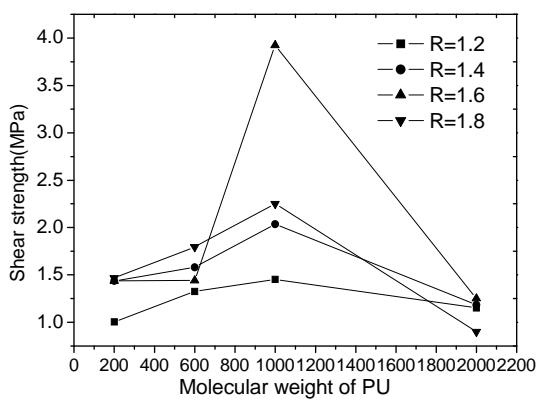

(c)

Figure 1. (a) The tensile strength; (b) The elongation at break (\%); and (c) The shear strength of IPN with different -NCO/-OH ratios in PU network.

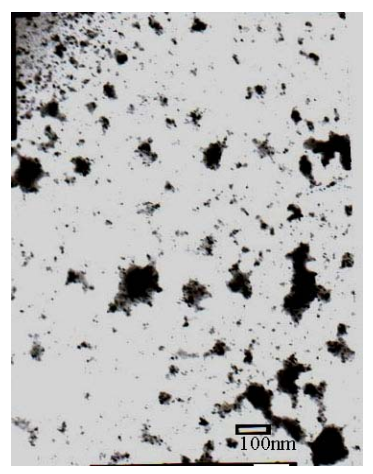

(a)

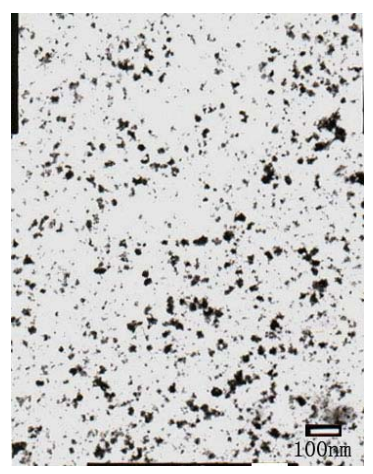

(b)

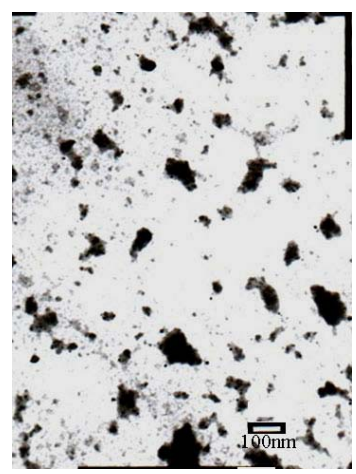

(c)

Figure 2. TEM photographs of 60/40 IPN with different molecular weight of PEGPU. (a) PEGPU600/UP IPN; (b) PEGPU1000/UP IPN; (c) PEGPU2000/UP IPN. 
and PEGPU2000/UP IPN. The better miscibility between the two phases of PEGPU1000 and UP in IPN might due to the higher hydroxyl value of PEGPU1000 and thus lead to the quicker react rate with the isocyanate group to form the PU network, and further lead to a little phase separation with UP phase. After the formation of the first component, the entropy in the system decreases, and the Gibbs free energy increases. Thus the network formed later becomes the filler of the first one. By adjusting the soft/hard segments ratios of different types of PEGPU in IPN, the phase separation could be controlled in some extent. The TEM results are thorough consistency of the mechanical detection results.

\subsection{The Effect of the PU/UP Component Ratios in IPN}

The component ratio of PU to UP is also the main factor that affects the compatibility of IPN. Figure 3 shows the tensile strengths, the elongation at break and the shear strengths of IPN with different PU to UP ratios. All the detection values increased at first and then decreased.

The results indicated that the appropriate crosslink density of PU and the appropriate penetrating degree of PU to UP for 60/40 IPN by using PEGPU1000 gave relative higher mechanical properties. And both the higher values of the strengths and the elongations of 60/40 PEGPU1000/ UP IPN, compared with the ordinary polymers and the each component network (PU and UP), also indicated the existence of such effects as mutual entanglement, forced compatibility, synergism of the networks during IPN's formation. The existence of the interpenetration and the entanglement molecular structures in IPN contributes the reinforced effect when the materials are elongated and thus improves the mechanical properties greatly than that of the ordinary materials. These effects provide a valid way to improve the materials' properties from the viewpoint of the selection of the components in IPN and the adjustment of the blending techniques. Further, these effects have great significance in obtaining the functional materials with outstanding properties.

Figure 4 shows the TEM photographs of IPN with different PU/UP component ratios. The higher PU content in IPN forms the first network and then becomes the continuous phase with UP appears as the dispersed phase. Seen from this figure, both 70/30 and 60/40 PEGPU1000/UP IPN show homogeneous microstructures with small domain sizes within nanometer scales. The higher UP amount in IPN leads to the more continuous degree of the dispersed phase with much finer microstructures.

\subsection{The Effect of the Amounts of $\mathrm{BaTiO}_{3}$ Nanopowders Added in IPN}

The previous studies $[10,11]$ have indicated that, the introduction of the inorganic filler into IPN can add the functional properties by the inorganic component, but meanwhile increase the phase separation to the system obtained. And the lower degree of the phase miscibility might further decrease the materials' mechanical properties. Figure 5 shows the tensile strength and the elongation at break of various -NCO/-OH values of IPN with different amounts of $\mathrm{BaTiO}_{3}$ nanopowders added. The values of the tensile strengths decreased with the increase of $\mathrm{BaTiO}_{3}$ nanopowders in IPN, but the values of the elongation at break displayed an opposite trend. The decreased tensile strengths might due to the less miscibility between the two apparent different modulus of $\mathrm{BaTiO}_{3}$ and IPN. And the increased elongations inferred that the little amount of filler can act as the plasticizer to IPN-based system.

The TEM photographs of the different amount of $\mathrm{Ba}-$ $\mathrm{TiO}_{3}$ nanopowders added in IPN are shown in Figure 6. The nanopowders are dispersed with some aggregations, but mostly with the original structures in the matrix of polymers. Larger $\mathrm{BaTiO}_{3}$ amount added into led to the appearance of higher aggregation.

\subsection{The Effect of the Component Sequences and time Intervals for Gradient IPN}

Previous studies also have shown that [11] the large amount

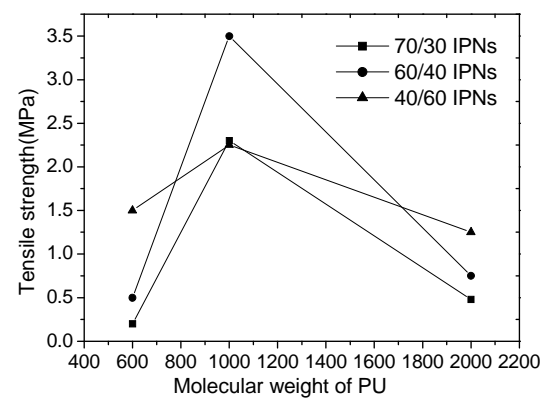

(a)

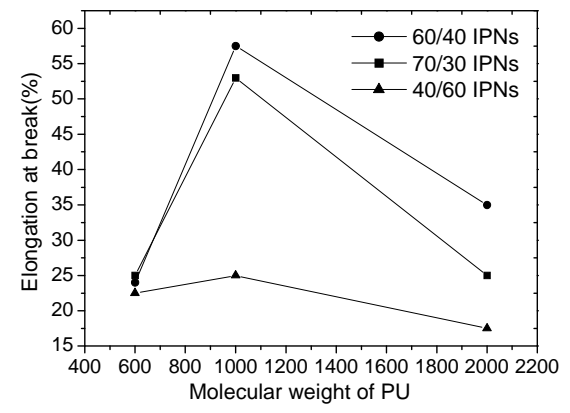

(b)

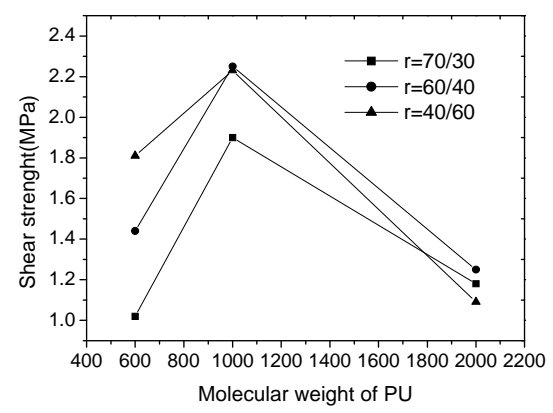

(c)

Figure 3. (a) The tensile strength; (b) The elongation at break (\%); and (c) The shear strength of IPN with different PU/UP component ratios. 


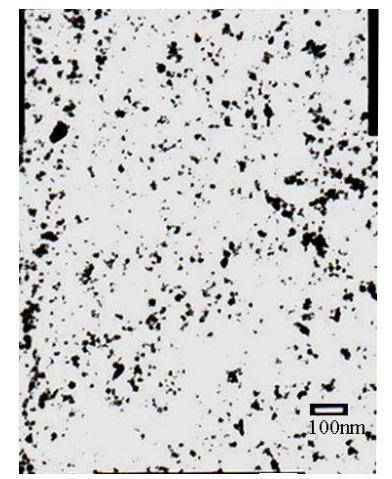

(a)

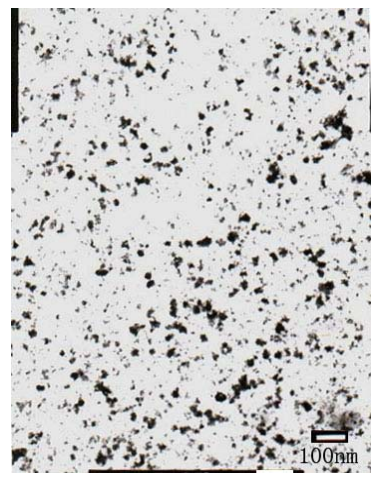

(b)
Figure 4. TEM photographs of IPN with different PU/UP component ratios. (a) 70/30 IPN; (b) 60/40 IPN.

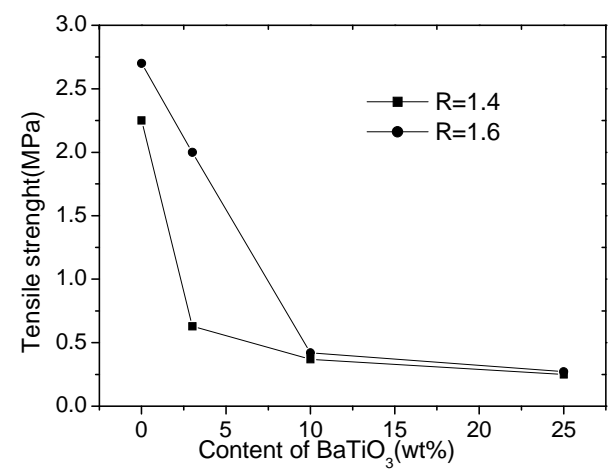

(a)

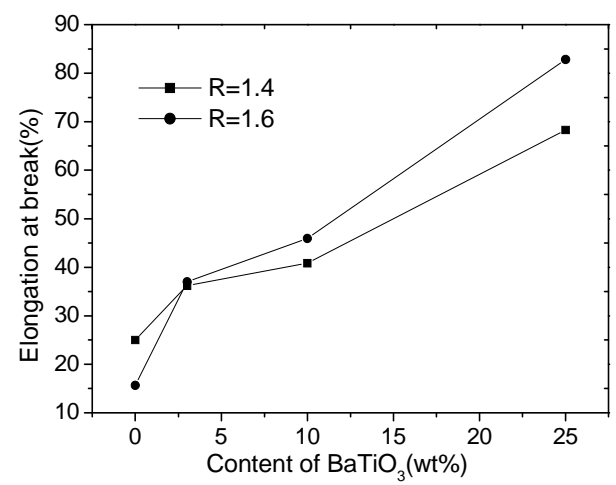

(b)

Figure 5. (a) The tensile strength and (b) Elongation at break (\%) of IPN/BaTiO ${ }_{3}$ composites with different $\mathrm{BaTiO}_{3}$ nanopowders added.

of the dual-entanglement structures formed during the individual IPN's formation by gradient techniques can improve the compatibility between the two obvious thermodynamic immiscibility networks. So the gradient technique can thus contribute the improved properties to IPN. Figure 7 shows the tensile strengths and the elongations at break of gradient IPN with different component ratio sequences at time intervals of $3.5 \mathrm{~h}$ during the curing process of the individual IPN. The higher modulus of UP (a)

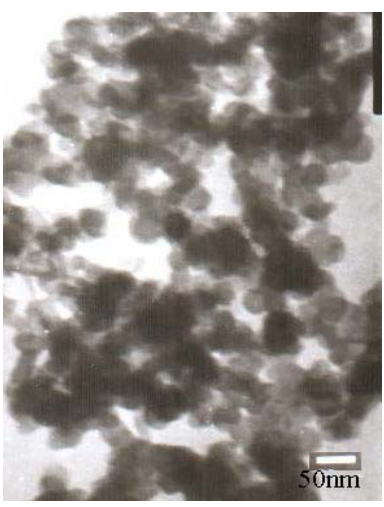

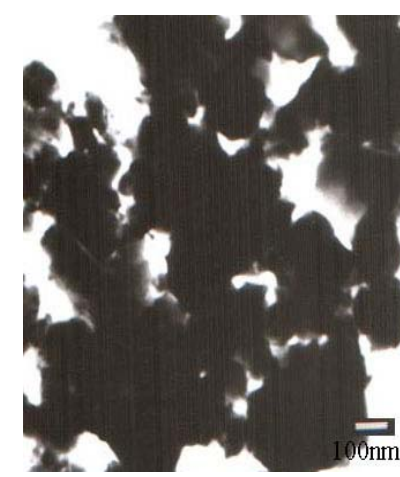

(b)
Figure 6. TEM photographs of IPN with different amounts of $\mathrm{BaTiO}_{3}$ nanopowders. (a) $3 \%$ (wt \%); (b) $10 \%$ (wt \%).

in gradient IPN contributes the better mechanical properties. Furthermore, from the comparison results with that of in Figure 2(a) of IPN, the gradient technique do improves the elastic property of IPN greatly. Also seen from the Figure 7, the larger amount of UP in gradient IPN with different components ratio sequences contributes relative higher tensile values and lower elongation values. For the gradient IPN with 40/60 - 60/40 - 70/30 components ratio sequences, the higher tensile values obtained without decrease much elongation values indicate that, novel materials with much more better mechanical properties can be designed by forming gradient structures within the different individual IPN.

Figure 8 shows the strain and the stress values of the $60 / 40$ - 70/30 - 80/20 gradient IPN with different time intervals. Seen from Figure 8, the gradient IPN with the time intervals of $3.5 \mathrm{~h}$ can contributes better mechanical properties. Both the higher tensile strength and the elongation at break proved the synergistic action of the more interpenetration in gradient IPN.

The TEM photographs of 40/60 - 60/40 - 70/30 gradient IPN with different time intervals are shown in Figure 9. Seen from this figure, compared with the individual IPN, the gradient IPN show the much finer and the homogeneous structures between the two phases with improved dual-continuity degree owing to the large amount of interpenetrating structures between two networks and transitional regions among phases.

\section{Conclusion}

The soft to hard segment ratio in PU, the component ratio of PU to UP in IPN, the $\mathrm{BaTiO}_{3}$ adding amount and the gradient technique all have effects to the compatibility and the mechanical properties of the IPN composed of the poly(ethylene glycol urethane) (PEGPU, with different molecular weight) and the unsaturated polyester resin 


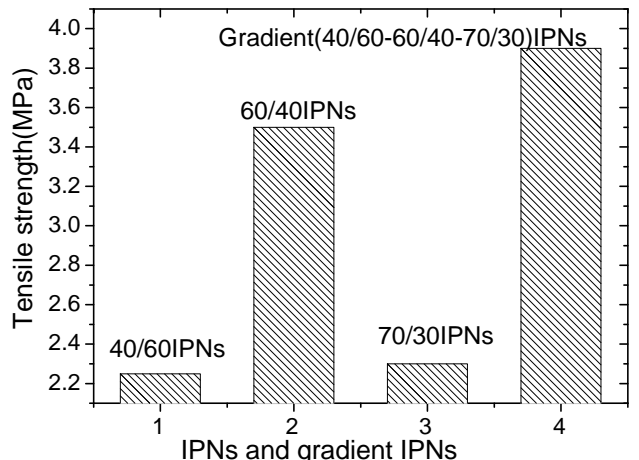

(a)

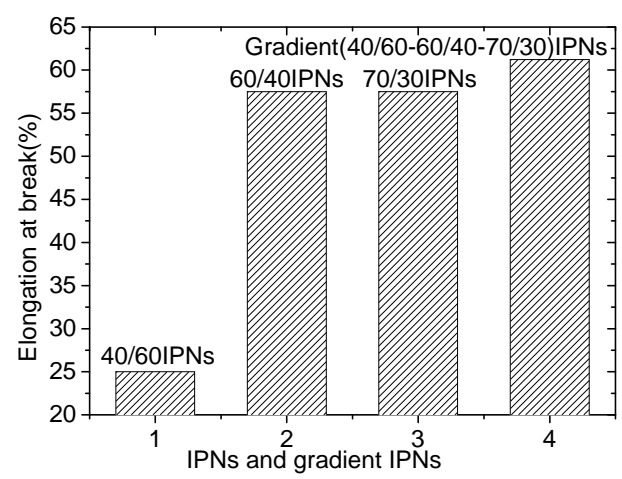

(b)

Figure 7. (a) The tensile strength and (b) The elongation at break (\%) of IPN and gradient IPN.

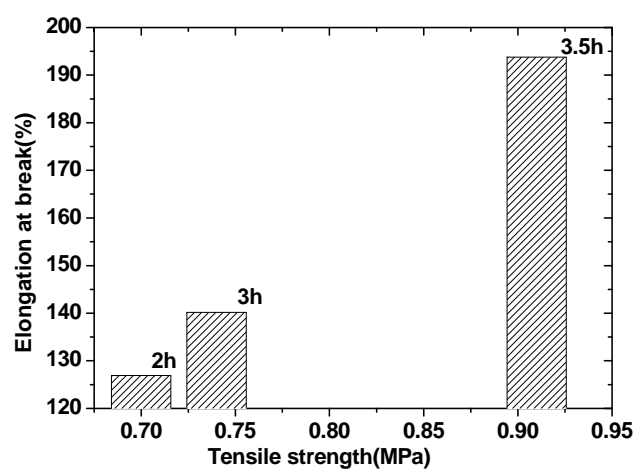

Figure 8. The tensile strength and the elongation at break (\%) of gradient IPN (40/60 - 60/40 - 70/30) with different time intervals.

(UP). The higher hydroxyl value of PEGPU in PU network can react faster than that of the other PEGPU. The appropriate crosslink density in IPN and the gradient structure can generate relative smaller domain sizes (within nanometer ranges). The relationship study of the compatibility and the mechanical properties shows that the better compatibility between the two networks in IPN and in filled IPN contributes both the improved strength and the elongation values. Moreover, the gradient structures can also increase the tensile strength and the elongation at break

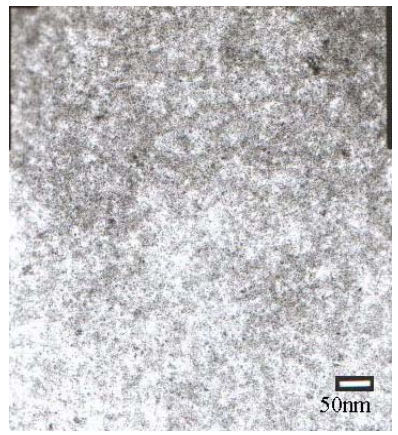

(a)

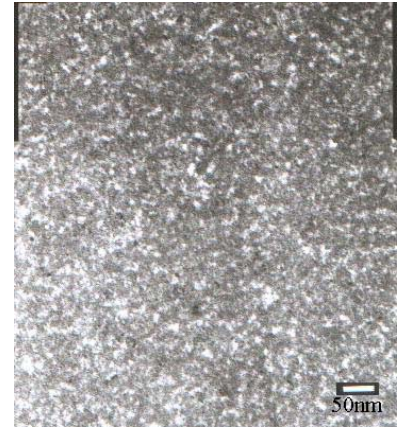

(b)
Figure 9. TEM photographs of 40/60 - 60/40 - 70/30 gradient IPN with different time intervals. (a) $3.5 \mathrm{~h}$; (b) $3.0 \mathrm{~h}$.

of IPN greatly compared with the each individual IPN, owing to the larger transitional ranges created by the gradient technique.

\section{Acknowledgements}

This work was supported by the Program for New Century Excellent Talents in University of China (NCET08-0165).

\section{REFERENCES}

[1] K. H. Hsieh, J. S. Tsai and K. W. Chang, "Interpenetrating Polymer Network of Polyurethane and Unsaturated Polyester: Mechanical Properties,” Journal of Materials Science, Vol. 26, No. 21, 1991, pp. 5877-5882. doi:10.1007/BF01130128

[2] T. Trakulsujaritchok and D. J. Hourston, "Damping Characteristics and Mechanical Properties of Silica Filled PUR/PEMA Simultaneous Interpenetrating Polymer Networks," European Polymer Journal, Vol. 42, No. 11, 2006, pp. 2968-2976. doi:10.1016/j.eurpolymj.2006.07.028

[3] M. A. Syed, Siddaramaiah and B. Suresha, "Mechanical and Abrasive Wear Behavior of Coleus Spent Filled Unsaturated Polyester/Polymethyl Methacrylate Semi Interpenetrating Polymer Network Composites," Journal of Composite Materials, Vol. 43, No. 21, 2009, pp. 23872400. doi:10.1177/0021998309341850

[4] S. C. Kim, D. Klempner and K. C. Frisch, "Polyurethane Interpenetrating Polymer Networks. I. Synthesis and Morphology of Polyrurethane-Poly(methyl Methacrylate) Interpenetrating Polymer Networks," Macromolecules, Vol. 9, No. 2, 1976, pp. 258-263. doi:10.1021/ma60050a016

[5] Y. L. Cai, P. S. Liu and X. B. Hu, "Microstructure-Tensile Properties Relationships of Polyrurethane/ Poly(urethane-Modified Bismaleimide) Interpenetrating Polymer Networks,” Polymer, Vol. 41, No. 15, 2000, pp. 5653-5660. doi:10.1016/S0032-3861(99)00797-1

[6] C. Vancaeyzeele, O. Fichet, J. Laskar, S. Boileau and D. Teyssie, "Polyisobutene/Polystyrene Interpenetrating Poly- 
mer Networks: Effects of Network Formation Order and Composition on the IPN Architecture,” Polymer, Vol. 47, No. 6, 2006, pp. 2046-2060. doi:10.1016/j.polymer.2006.01.026

[7] D. Y. Tang, X. L. Wu and L. S. Qiang, "Polymerization Process and Morphology of PU/VER IPNs,” Journal of Materials Science \& Technology, Vol. 23, 2007, pp. 423426.

[8] F. J. Hua and C. P. Hu, "Interpenetrating Polymer Networks of Epoxy Resin and Urethane Acrylate Resin: 2. Morphology and Mechanical Property," European Polymer Journal, Vol. 36, No. 1, 2000, pp. 27-33. doi:10.1016/S0014-3057(99)00027-0

[9] K. H. Hsieh, J. L. Han and C. T. Yu, “Graft Inperpenetrating Polymer Networks of Urethane-Modified Bismaleimide and Epoxy(I): Mechanical Behavior and Morphology,” Polymer, Vol. 42, No. 6, 2001, pp. 2491-2500. doi:10.1016/S0032-3861(00)00641-8

[10] D. Y. Tang, C. L. Qin and W. M. Cai, "Preparation, Morphology, and Mechanical Properties of Modified-PU/ UPR Graft-IPN Nanocomposites with $\mathrm{BaTiO}_{3}$ Fiber," Materials Chemistry and Physics, Vol. 82, No. 1, 2003, pp. 73-77. doi:10.1016/S0254-0584(03)00162-7

[11] D. Y. Tang, J. S. Zhang, D. R. Zhou and L. C. Zhao, "Influence of $\mathrm{BaTiO}_{3}$ on Damping and Dielectric Properties of Filled Polyurethane/Unsaturated Polyester Resin In- terpenetrating Polymer Networks,” Journal of Materials Science, Vol. 40, No. 13, 2005, pp. 3339-3345. doi:10.1007/s10853-005-0423-3

[12] J. Yang and M. A. Winnik, "Polyurethane-Polyacrylate Interpenetrating Networks. 1. Preparation and Morphology,” Macromolecules, Vol. 29, No. 22, 1996, pp. $7047-$ 7054. doi:10.1021/ma9601373

[13] L. Zhang and Q. Zhou, "Effects of Molecular Weight of Nitrocellulose on Structure and Properties of Polyurethane/Nitrocellulose IPN,” Journal of Polymer Science: Part B: Polymer Physics, Vol. 37, No. 14, 1999, pp. 1623-1631.

doi:10.1002/(SICI)1099-0488(19990715)37:14<1623::AI D-POLB7>3.0.CO;2-5

[14] Y. S. Lipatov, V. F. Rosovitsky, T. T. Alekseeva and N. V. Babkina, "Effect of Physical Ageing on the Viscoelasticity on Interpenetrating Polymer Networks," Polymer International, Vol. 49, No. 4, 2000, pp. 334-336. doi:10.1002/(SICI)1097-0126(200004)49:4<334::AID-PI 371>3.0.CO;2-Q

[15] K. H. Yoon, S. T. Yoon and O. O. Park, "Damping Properties and Transmission Loss of Polyurethane. I. Effect of Soft and Hard Segment Compositions," Journal of Applied Polymer Science, Vol. 75, No. 5, 2000, pp. 604-611. doi:10.1002/(SICI)1097-4628(20000131)75:5<604::AIDAPP2>3.0.CO;2-\# 